\title{
Dissociative electron attachment to abasic DNA
}

\author{
Sylwia Ptasińska* and Léon Sanche
}

\author{
Received 15th November 2006, Accepted 18th January 2007 \\ First published as an Advance Article on the web 12th February 2007 \\ DOI: $10.1039 / b 616619 a$
}

\begin{abstract}
Thin films of the short single DNA strand, GCAT, in which one of the bases has been removed were bombarded with 3 to $15 \mathrm{eV}$ electrons. The yield functions of the $\mathrm{H}^{-}, \mathrm{O}^{-}$and $\mathrm{OH}^{-}$ions desorbed from these films exhibit a broad peak near $9 \mathrm{eV}$, which is attributed to dissociative electron attachment to the basic molecules. Whereas removal of any one of the bases considerably decreases $N$-glycosidic and backbone $\mathrm{C}-\mathrm{O}$ bond scission, the creation of basic sites does not appreciably modify bond rupture leading to anion electron stimulated desorption. These seemingly contradictory results make it possible to propose a detailed mechanism leading to the transfer of electrons in the range 5-13 eV within DNA.
\end{abstract}

\section{Introduction}

Following the discovery that bond scission in DNA produced by electrons of less than $15 \mathrm{eV}$ occurs essentially via the formation of transient anions, ${ }^{1}$ considerable research has been performed to determine the precise mechanism and location of the damage. ${ }^{2}$ Relevant experiments have included mass spectrometry and high resolution electron energy loss investigations of the basic molecular components of DNA in their gaseous and condensed phases, as well as chemical analysis of the products resulting from low-energy electron (LEE) bombardment of lyophilized DNA and self assembled monolayers (SAM) of the molecule. ${ }^{2}$ By comparing the results generated by these various experiments, it has been possible in many cases, to deduce the mechanism responsible for a specific bond scission. It is now established that below $15 \mathrm{eV}$, electrons can break DNA bonds via dissociative electron attachment (DEA) with or without concomitant formation of small stable anions and radicals from DNA films. Production of $\mathrm{O}^{-}$occurs via the temporary localization of $9.2 \mathrm{eV}$ electrons on the $\pi^{*}$ double bond of the phosphate group, ${ }^{3}$ whereas $\mathrm{OH}^{-}$desorption from DNA films is caused by the localization of 5.5 and $6.7 \mathrm{eV}$ electrons on the protonated form of the phosphate group. ${ }^{4}$ Stimulated desorption of $\mathrm{H}^{-}$occurs as the result of temporary capture of $8-12 \mathrm{eV}$ electrons on the bases with a small contribution from the sugar group. ${ }^{3}$ Strand breaks are essentially due to temporary electron localization on the phosphate group followed by scission of the $\mathrm{C}-\mathrm{O}$ bond at the $3^{\prime}$ or $5^{\prime}$ positions. $^{5-8}$ Base release is caused by resonance decay into dissociative electronic excitation and/or DEA channels. ${ }^{7-9}$

Due to the possibility of non-thermal electron transfer within DNA, the basic component (or components), that initially captures the electron in a resonance state is not necessarily the one that dissociates due to the presence of the additional electron. ${ }^{8}$ According to several theoretical studies

Groupe en Sciences des Radiations, Faculté de Médecine, Université de Sherbrooke, Sherbrooke, QC, Canada J1H 5N4. E-mail:

sylwia.ptasinska@usherbrooke.ca below $3 \mathrm{eV}$, electrons cleave the $\mathrm{CO}$ bond of the DNA backbone at the $3^{\prime}$ and $5^{\prime}$ positions, not only via direct capture by a phosphate group, ${ }^{6}$ but also via electron transfer. ${ }^{5,10-12}$ In brief, an incoming electron captured by one of the lowest $\pi^{*}$-resonance states of the bases transfers to the sugarphosphate group of the molecule where it resides for a sufficiently long time to cause $\mathrm{C}-\mathrm{O} \sigma$ bond rupture. This latter process may also be induced by proton transfer to a negatively charged base during the lifetime of a resonance. ${ }^{13}$ Such a transfer would leave an extra electron on the sugar or phosphate unit, which could also lead to rupture of the sugar-phosphate CO bond, again via DEA to the phosphate unit. Thus, according to present theoretical calculations $\mathrm{C}-\mathrm{O}$ bond cleavage in DNA occurs as a consequence of DEA to the phosphate group, but the extra electron forming the local transient anion may come from outside DNA or via electron and proton transfer within the molecule.

The hypothesis of electron transfer from a base to the phosphate group was first supported experimentally by measurements of SSB in plasmid DNA below $5 \mathrm{eV} .{ }^{14}$ Later, Zheng et al. bombarded thin molecular films of a short single strand of DNA, with electrons of energies between 4 and $15 \mathrm{eV}{ }^{8}$ By high-pressure liquid chromatography, they identified 12 fragments of the oligonucleotide GCAT, whose nomenclature is shown on the right of Scheme 1, to be sequentially composed of the bases guanine $(\mathrm{G})$, cytosine $(\mathrm{C})$, adenine $(\mathrm{A})$ and thymine (T). The yield functions exhibited maxima at 6 and 10-12 eV, which were interpreted as due to the formation of transient anions leading to fragmentation. Below $15 \mathrm{eV}$, these resonances dominated the bond dissociation processes. All four non-modified bases were released from the tetramer, by cleavage of the $N$-glycosidic bond, which occurs principally via the formation of core-excited resonances located around 6 and $10 \mathrm{eV}$. The formation of the other non-modified products was found to be due to strand breaks caused by DEA directly to the phosphate unit or via electron transfer from the bases. With regard to the latter mechanism, Zheng et al. ${ }^{8}$ concluded that both shape and core-excited resonances are formed by electron attachment to the bases. The latter resonance would 


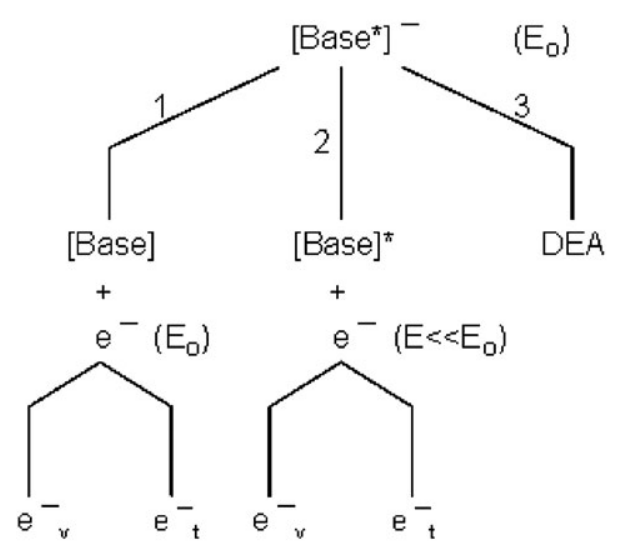

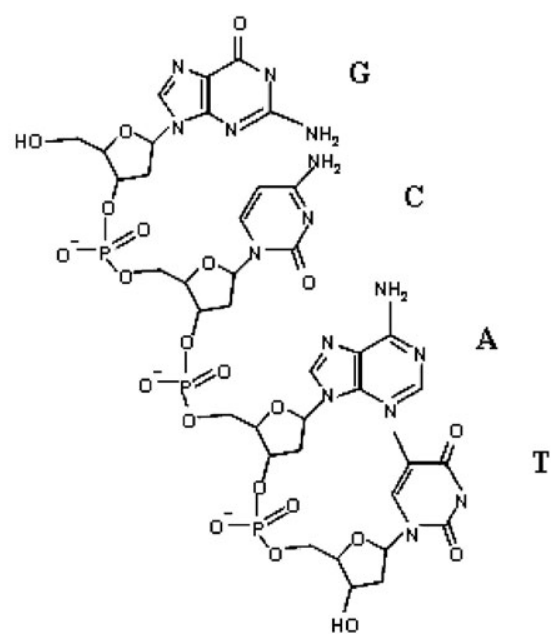

Scheme 1

retain the electron for a sufficiently long period to allow dissociation of the $N$-glycosidic bond. Shape resonances, which are known to be much shorter-lived beyond $\sim 5 \mathrm{eV}$, were not expected to contribute to dissociation, but according to the authors would be responsible for electron transfer to the phosphate group, leading to the formation of a local shape or core-excited resonance on that group.

Zheng et al. ${ }^{15}$ repeated these experiments with abasic forms of the tetramer GCAT, i.e., XCAT and GCXT, where X represents a base which has been removed and replaced by a hydrogen atom. Their results showed that at $6 \mathrm{eV}$, and possibly below, electrons break the DNA backbone almost exclusively via electron transfer, whereas at higher energy direct electron attachment to the phosphate group contributes to strand scission. Furthermore, base release induced by $6-12 \mathrm{eV}$ electrons was found to be strongly affected by the presence of an abasic site; in both XCAT and GCXT, the yield of detached bases was found to be up to an order of magnitude smaller than that from GCAT. Thus, the electron initial capture amplitude was suggested to be highly sensitive to the number and possibly the geometrical arrangement of the bases, indicating the presence of a strong collective effect. In fact, in recent model calculations $^{16,17}$ it has been shown that, because of its wavelength being longer than the inter-unit distances in DNA at low energies $\left(E_{0} \leq 6 \mathrm{eV}\right)$, the electron scattered within DNA has a high probability of being delocalized in the molecule. Thus, the interacting electron is first likely to undergo multiple intersite scattering before being captured at a specific basic unit in a resonant state. As shown theoretically, the partial wave content of the electron wavefunction inside DNA then becomes dominated by constructive interferences, which enhance considerably (i.e., up to at least an order of magnitude) the capture probability on all basic units, including the bases. ${ }^{18}$ It is, therefore, highly probable that according to their mechanism there exists in GCAT a strong coherence enhancement of the initial electron wavefunction owing to the periodicity in the positions of the bases; but when this periodicity is broken in GCXT and XCAT, the magnitude of the base release decreases considerably (i.e. up to an order of magnitude). Similar, but smaller, coherence effects were also observed in the yield of products corresponding to strand breaks.

In the present work, we test the validity of these basic mechanisms proposed to explain electron transfer within DNA by investigating anion desorption induced by $3-15 \mathrm{eV}$ electrons on thin films of the abasic tetramers XCAT, GXAT, GCXT and GCAX. Contrary to the results of Zheng et al. ${ }^{15}$ no strong collective effects are found in the anion signals arising from DEA to these tetramer. These findings raise an important question: how can DEA leading to bond rupture in DNA be subjected to strong coherence effects when no such coherence is observed in the anion yield signal resulting from DEA to the same forms of DNA. To answer this question, we have to provide a model of electron transfer valid for all electron energies below $15 \mathrm{eV}$ and refine previously suggested mechanisms leading to strand breaks and base release. The proposed electron capture and transfer mechanism is shown to be consistent with all experimental and theoretical data presently available.

\section{Experimental}

The apparatus used in the present investigation has been described in detail elsewhere. ${ }^{19,20}$ Only a brief description of the procedure and experimental arrangement is given here. The abasic tetramers were purchased from Alpha DNA (Montreal, QC) and purified by high performance liquid chromatography. Each sample was dissolved in $80 \mu \mathrm{l}$ of sterile deionized (Millipore) water, deposited on the chemically clean tantalum substrate, over an area of $1.3 \mathrm{~cm}^{2}$ and frozen at liquid nitrogen temperature. It should be mentioned that the DNA solution was prepared without any added salt, so that the negative charge on one of the oxygens of the phosphate group is counterbalanced mainly by a proton $\left(\mathrm{H}^{+}\right.$from $\left.\mathrm{H}_{2} \mathrm{O}\right)$. The sample was then lyophilized with a hydrocarbon-free sorption pump at 5 mTorr. After lyophilization, samples were exposed to the atmosphere for about $10 \mathrm{~min}$ and placed in a sample holder which was inserted in a load-lock vacuum system $\left(\sim 1 \times 10^{-8}\right.$ Torr $)$. After evacuation for $12 \mathrm{~h}$, the samples were transferred to an ultrahigh vacuum (UHV) 
chamber $\left(\sim 2 \times 10^{-10}\right.$ Torr $)$, one at a time, via a gate valve. Once in the UHV system, the surface of the DNA sample was positioned perpendicular to a mass spectrometer (Extrell 150QC). An electron beam produced by a Kimball Physics ELG-2 gun, with an energy resolution of $0.5 \mathrm{eV}$, was focused on a $2 \mathrm{~mm}^{2}$ spot on the sample, at an incident angle of $70^{\circ}$ to the surface normal. The average thickness of the film was estimated from the volume of a deposited DNA solution at a known solid density of $1.7 \mathrm{~g} \mathrm{~cm}^{-3} \cdot{ }^{21}$ All results presented in this work were performed on films of $1.6( \pm 0.3) \mathrm{nm}$ thickness. The average film thickness is smaller than the effective range (12-14 nm) for damaging DNA with $10-50 \mathrm{eV}$ electrons ${ }^{22}$ and the penetration depth/mean free path $(15-30 \mathrm{~nm})$ of $5-100 \mathrm{eV}$ electrons in liquid water or amorphous ice. ${ }^{23}$ Thus, most electrons from the beam are transmitted through the film of oligonucleotides under single scattering conditions. In the present experiments, the sample at a fixed position was irradiated by electrons with incident energies of between 3 and $15 \mathrm{eV}$ and a maximum transmitted current of $10 \mathrm{nA}$. The electron energy scale was calibrated by taking $0 \mathrm{eV}$ as the onset of electron transmission through the film, with an estimated error of about $\pm 0.3 \mathrm{eV} .^{24}$ The resulting ion yield functions were repeatable to within $10 \%$. All recorded ion yields were the averages of two successive electron energy scans, averaged over at least three new films.

\section{Results}

The yields of $\mathrm{H}^{-}, \mathrm{O}^{-}$and $\mathrm{OH}^{-}$for all abasic forms of the GCAT tetramer are presented in Fig. 1-3, respectively, and compared to previous data obtained from similar GCAT films. The baselines of anion yield functions for abasic oligonucleotides have been shifted vertically for clarity in all figures. The $\mathrm{CN}^{-}$and $\mathrm{CNO}^{-}$anions were also observed, but owing to a very weak signal, the energy dependence of their yields is not presented here. The broad peak having a maximum near $9.2 \mathrm{eV}$ is due to DEA, which is initiated by the resonant capture of an electron to the molecule to form a dissociative core-excited transient anion state. ${ }^{2,25}$

Heavier mass anions ( $>42 \mathrm{amu}$ ) have not been observed, presumably due to their insufficient kinetic energy to overcome the attractive polarization and charge image forces induced by the anion in the molecular film and the metal substrate. ${ }^{26}$ All fragment anions detected from the abasic tetramers have also been observed in the studies of DEA to isolated nucleobases in the gas phase (i.e. guanine, ${ }^{27}$ cytosine, ${ }^{28,29}$ adenine, ${ }^{27,30}$ thy-

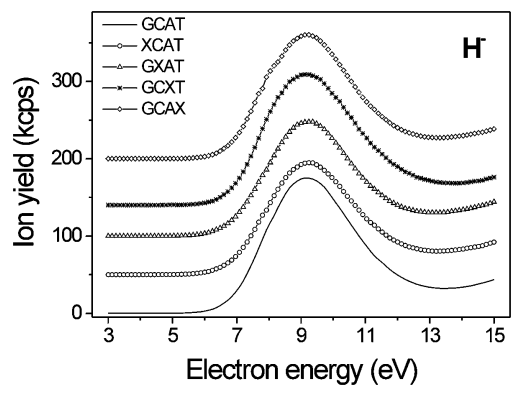

Fig. 1 The ion yield of $\mathrm{H}^{-}$from GCAT tetramer and its abasic forms.

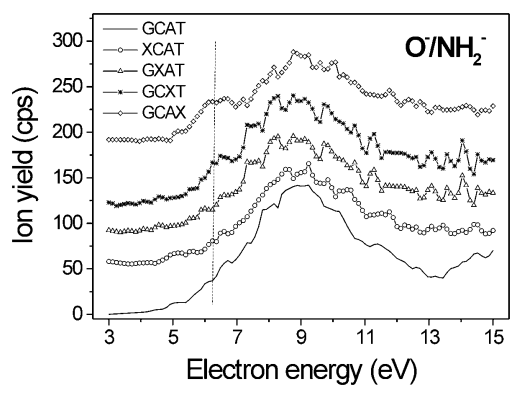

Fig. 2 The ion yield of $\mathrm{O}^{-} / \mathrm{NH}_{2}{ }^{-}$from GCAT tetramer and its abasic forms.

mine $^{28-33}$ ) and the hydroxyl anion from the phosphate group. ${ }^{34}$ Possible cleavage pathways for GCAT have recently been described in experiments, where anion yield functions obtained from films of condensed GCAT were compared with gas-phase data. ${ }^{25}$ In the present experiment, the $\mathrm{H}^{-}$signal (Fig. 1) is the most abundant of all anions desorbed from the abasic tetramer films.

From a previous comparison of gas-phase data with those from GCAT, the pronounced peak at $9.2 \mathrm{eV}$ was ascribed to $\mathrm{H}^{-}$production at the carbon sites of nucleobases. ${ }^{25}$ In all cases, the shape of the ion yield functions in Fig. 1 are almost identical, including the positions of their maxima; differences appear only in the peak intensities. The efficiencies of the DEA process observed for GCAT and GCXT tetramers are equal within the estimated experimental uncertainty of about $10 \%$. This finding is in good agreement with results obtained from gas phase studies, which indicate that $\mathrm{H}^{-}$formation at the electron energy of $9.2 \mathrm{eV}$ is not efficient for adenine. ${ }^{30}$ Thus removing of adenine from the oligos does not decrease the $\mathrm{H}^{-}$yield significantly. Dependence of $\mathrm{H}^{-}$ion yields on the electron energy is the same for XCAT and GXAT within the error bars.

The yield of anions of mass $16 \mathrm{amu}$, shown in Fig. 2, can be attributed either to the formation of $\mathrm{O}^{-}$from the phosphate group of the backbone of DNA or to $\mathrm{O}^{-}$or $\mathrm{NH}_{2}{ }^{-}$from the nucleobases. In the case of thymine, ${ }^{28,29}$ this mass was ascribed to $\mathrm{O}^{-}$, since no amino group is present in the molecule. For cytosine $^{28,29}$ and guanine ${ }^{27}$ the assignment is ambiguous. It is worth noting that in spite of the presence of the amino group in adenine no anionic fragments of mass 16 were observed from electron impact on gaseous and condensed adenine. ${ }^{27}$ A priori, the magnitude of the resonant peaks in Fig. 2 is the

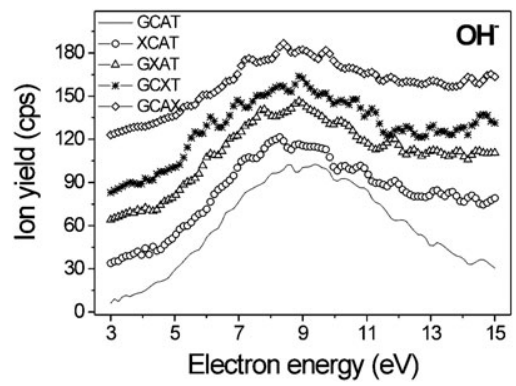

Fig. 3 The ion yield of $\mathrm{OH}^{-}$from GCAT tetramer and its abasic forms. 
Table 1

\begin{tabular}{lllr}
\hline & Form & Area (a.u) & $\%$ \\
\hline $\mathrm{H}^{-}$ & GCAT & $70 \times 10^{4}$ & 100 \\
& XCAT & $59 \times 10^{4}$ & 84 \\
& GXAT & $59 \times 10^{4}$ & 84 \\
& GCXT & $70 \times 10^{4}$ & 100 \\
& GCAX & $62 \times 10^{4}$ & 89 \\
$\mathrm{O}^{-}$ & & \\
& GCAT & 750 & 100 \\
& XCAT & 550 & 73 \\
& GXAT & 580 & 77 \\
& GCXT & 670 & 89 \\
$\mathrm{OH}^{-}$ & GCAX & 520 & 69 \\
& & & 100 \\
& GCAT & 720 & 89 \\
& XCAT & 640 & 82 \\
& GXAT & 610 & 65 \\
\hline
\end{tabular}

sum of signals at a mass of 16 amu arising from the nucleobases and the phosphate group.

The shape of the yield functions from the abasic tetramers resembles quite well that obtained from GCAT. ${ }^{25}$ However, a minor difference in the shape of the ion yield is discernible in the case of desorption of the 16 amu anion from the modified oligomers. A shoulder around $6 \mathrm{eV}$ observed from GCAT, which is marked by a vertical line in Fig. 2, is slightly suppressed in the case of XCAT and GXAT.

Desorption of $\mathrm{O}^{-}$induced by LEE impinging on a phosphate film $\left(\mathrm{NaH}_{2} \mathrm{PO}_{4}\right)$ has recently been reported. ${ }^{35}$ The $\mathrm{O}^{-}$ yield function exhibited a single broad peak at $8.2 \mathrm{eV}$ with the onset at approximately $4 \mathrm{eV}$. The formation of the $\mathrm{O}^{-}$fragment required a substantial energy $(\sim 3.31 \mathrm{eV})$, due to the large dissociation energy of the $\mathrm{P}=\mathrm{O}$ bond. The $\mathrm{NaH}_{2} \mathrm{PO}_{4}$ data corroborate the previous conclusion from LEE-DNA experiments, ${ }^{3}$ that within DNA, $\mathrm{O}^{-}$is formed by DEA to the phosphate group. The observed shift of $1 \mathrm{eV}$ in the yield maxima for $\mathrm{O}^{-}$from oligonucleotides and the phosphate salt can be due to the different molecular enviroment. From these considerations, we conclude that the $16 \mathrm{amu}$ signal is principally due to $\mathrm{O}^{-}$desorption arising from the $\mathrm{P}=\mathrm{O}$ bond of the phosphate group.

As seen in Fig. 3, the detection of $\mathrm{OH}^{-}$from the abasic tetramer films is observed over a wide range of electron energy (3-15 eV). Further measurements (not presented here) showed the threshold for the formation of this anion to lie around $2.5 \mathrm{eV}$. The shapes of the $\mathrm{OH}^{-}$yield functions shown in Fig. 3 are the same as that found for the corresponding anion from the non-modified tetramer. According to recent experiments, ${ }^{4,25}$ the source of $\mathrm{OH}^{-}$ions in the GCAT molecule arises from the phosphate group in the backbone of DNA and one of the terminal OH of a deoxyribose sugar.

Table 1 shows the integrated DEA peak intensities taken from the curves of Fig. 1-3. Taking the yields of $\mathrm{H}^{-}, \mathrm{O}^{-}$and $\mathrm{OH}^{-}$from GCAT as $100 \%$, the relative yields of these anions from the abasic tetramers are displayed in the rightmost column. As expected, removal of a base lowers the yields with respect to that from GCAT.

\section{Disscusion}

As seen from Table 1, anion desorption does not depend very much on the presence of an abasic site in GCAT. From a purely classical point of view, if the anion signals arose exclusively from initial electron attachment on a base and if each base were given an equal weight for producing these anion yields, we should observe anion signals from the abasic tetramers which are $75 \%$ of that for GCAT. For $\mathrm{H}^{-}$the signal averaged for all abasic tetramers is higher $(88 \%)$ than this value, whereas for $\mathrm{O}^{-}$it averages close to $75 \%$; for $\mathrm{OH}^{-}$the averaged signal diminished to $81 \%$. These results clearly indicate the absence of quantum mechanical or collective effects in the initial interaction of the electron with DNA (i.e. DEA yields are essentially directly proportional to the number of bases for $\mathrm{OH}^{-}$and $\mathrm{O}^{-}$desorption).

The yields, being on average remarkably higher than $75 \%$ for $\mathrm{H}^{-}$, possibly arise from the contribution from the sugar group which may not be much affected by the creation of an abasic site. In experiments with 40-base pair and plasmid DNA, the $\mathrm{H}^{-}$signal has been observed to arise from both the bases and the sugar group. ${ }^{3}$ On the other hand, these same experiments, as well as those performed with single and double stranded SAM of DNA have demonstrated that both the $\mathrm{O}^{-}$ and $\mathrm{OH}^{-}$signals arise from DEA to the phosphate group, by direct electron capture and/or via electron transfer. ${ }^{4}$ In our experiment, however, if such DEA processes arose only from direct attachment to the phosphate group, no significant decrease would be observed in the $\mathrm{O}^{-}$and $\mathrm{OH}^{-}$signals from electron impact on the abasic tetramers, unless removal of a base considerably modifies the resonance parameters on the phosphate group. Such a modification would increase or decrease $\mathrm{O}^{-}$desorption by, respectively, either increasing or decreasing the lifetime of the transient anion and/or the capture probability. Thus, predicting the magnitude of the change in $\mathrm{O}^{-}$desorption requires knowledge of these variables, which themselves depend on induced polarization, chemical bonding and molecular arrangement. ${ }^{2}$ Although it is not possible to estimate the possible change in $\mathrm{O}^{-}$yield caused by a modification of the resonance parameters of the phosphate transient anion upon abasic sites creation, there is no reason to believe that the calculation would give precisely a $25 \%$ decrease in $\mathrm{O}^{-}$desorption, as expected from the electron transfer mechanism and observed experimentally. We therefore suggest that electron transfer occurs from the bases to the phosphate group in the formation of $\mathrm{O}^{-}$and $\mathrm{OH}^{-}$via $\mathrm{DEA}$ of 5-12 eV electrons to DNA. Whereas $\mathrm{O}^{-}$almost exclusively arises from the double bonded oxygen of the phosphate group in long DNA chains, in the case of a small oligonucleotide like GCAT, contributions to the $\mathrm{OH}^{-}$signal can also arise from the $\mathrm{OH}$ group of the terminal bases. The slightly larger $\mathrm{OH}^{-}$ signal compared to the $\mathrm{O}^{-}$signal, seen from Table 1, indicates that the possibility of a contribution from direct DEA to the phosphate group cannot be eliminated.

The present results show obviously that the initial electron wave is not strongly affected by diffraction effects as recently proposed to explain the strong decrease in base release and strand breaks induced by $6-12 \mathrm{eV}$ electrons, upon creation of an abasic site in GCAT. 
In fact, according to theory, coherent enhancement of the wavefunction initially scattered within DNA is relatively modest at 9-10 eV, but below $4 \mathrm{eV}$ can reach one order of magnitude for $\ell=2$ partial waves and two orders of magnitude for $\ell=3$ partial waves. ${ }^{16-18}$ In general, as the electron energy decreases, the electron wavefunction becomes larger and more delocalized and hence diffraction, which is structure dependent, becomes prominent.

There exist two possible mechanisms by which an electron temporarily attached to a base can transfer to the phosphate group. One mechanism is quasi-elastic through bond transfer, by which the extra electron is re-emitted, without losing significant amount of energy, into a "conduction band" composed of an overlap of all extra electron states of the DNA molecule above the energy of the vacuum level. Consequently, this delocalized electron can relocalize on the phosphate group, where again it can form a shape or core excited resonance on that group. The other possibility is inelastic through bond transfer. ${ }^{15}$ In this case, the extra electron on the base undergoes the same transfer process, but leaves the base in an electronically excited state. The first process is the simplest, but in the present case requires a final anion state to exist near 9-10 eV on the phosphate group, be dissociative and live a sufficient time for the $\mathrm{C}-\mathrm{O}$ bond to break. At such high energies shape resonances are usually too short-lived to cause dissociation $^{36}$ and were not observed to dissociate the phosphate group analog $\mathrm{NaH}_{2} \mathrm{PO}_{4} \cdot{ }^{35}$ However, DEA from core excited resonances within the range $5-12 \mathrm{eV}$ was found to lead to $\mathrm{H}^{-}, \mathrm{O}^{-}$and $\mathrm{OH}^{-}$production from $\mathrm{NaH}_{2} \mathrm{PO}_{4}{ }^{35}$

The second mechanism is the one proposed by Zheng et al. ${ }^{15}$ to explain $\mathrm{C}-\mathrm{O}$ bond scission in the DNA backbone induced by 6-eV electrons via electron transfer. They argued from LEE energy-loss spectroscopy experiments on the DNA bases that since electronically excited states exist within the 3.5 to $6 \mathrm{eV}$ range, ${ }^{37,38}$ excitation of these states by $6 \mathrm{eV}$ electrons forming a shape or core-excited resonance on a base would liberate electrons of 2.3, 2 and $1.1 \mathrm{eV}$ which could then transfer to the backbone. Thus, electron transfer from a DNA base $\pi^{*}$ to a $\mathrm{C}-\mathrm{O} \sigma^{*}$ orbital would occur at the predicted energies (i.e., below $3 \mathrm{eV}) .{ }^{10}$ In our case, the incident $9-10 \mathrm{eV}$ electrons could also electronically excite a base and by exciting higher energy electronic states liberate electrons within the $0-3 \mathrm{eV}$ range. However, since this process would be the same as that proposed by Zheng et al., ${ }^{15}$ it should also create a strong decrease in our DEA signal upon creation of an abasic site. This phenomenon is not observed in Fig. 1-3 and we therefore consider the possibility that the $9-\mathrm{eV}$ resonance decays more easily in the "elastic" channel so that our $\mathrm{O}^{-}$and $\mathrm{OH}^{-}$signals arise principally from quasi-elastic electron transfer. In this case, since coherent enhancement of the wavefunction of a $9 \mathrm{eV}$ electron by base stacking is much smaller than for a 0-3 eV electron, we expect only a small decrease in the magnitude of the $\mathrm{O}^{-}$and $\mathrm{OH}^{-}$signals, upon removal of a base, as observed experimentally. We therefore proposed that the 9-eV resonance in our experiment decays by direct DEA to the bases to produce the $\mathrm{H}^{-}$signal and by quasi-elastic electron transfer to produce the major portion of the $\mathrm{O}^{-}$and $\mathrm{OH}^{-}$yields. In our previous explanation on strand breaks and base release, we assumed that electron diffraction occurred along the base stack before electron capture. Now, we understand from the present experiments and theory ${ }^{16}$ that amplification of strand breaks and base release, via constructive interference occurs preferentially at very low electron energies. Thus, in the $\mathrm{N}$-glycosidic and $\mathrm{C}-\mathrm{O}$ bond scission process, the electron can first be captured by a base to form a core-excited resonance, which releases a very low energy (0-4 eV) electron; the latter's wavefunction can be coherently enhanced and can lead to a strong amplification of bond rupture, as previously reported. ${ }^{15}$

\section{Conclusions}

After considering the present results and the findings from previous studies on longer DNA molecules in the single and double stranded configurations, ${ }^{1-4}$ the chemical analysis of the products formed by LEE impact on GCAT, GCXT and $\mathrm{XCAT}^{7,8,15}$ and various theoretical calculations, ${ }^{5,6,10-12,39}$ we now propose a single model of resonance interaction of LEE with DNA. As shown in the left part of Scheme 1, the incoming electron forms first a core-excited ${ }^{40}$ or core-excited shape resonance on a base at an energy $E_{0}$ close to or above the first electronic excitation energy threshold. The transient anion can decay into three channels: (1) the elastic channel where the electron is reemitted with the same energy $\left(E_{0}\right) ;(2)$ the electronically inelastic channel which leads to electronic excitation of a base and the release of a very low energy ( $E=0-4 \mathrm{eV}$ ) electron; and (3) the DEA channel which fragments the parent base. In cases (1) and (2), the electron can be re-emitted into the vacuum $\left(\mathrm{e}_{\underline{v}}^{-}\right)$or transfer $\left(\mathrm{e}_{\underline{t}}^{-}\right)$within DNA. The branching ratios between these possibilities depend on the magnitude of the departing electron wavefunction in vacuum and within DNA. As mentioned previously, owing to internal diffraction, the magnitude of the square of the electron wavefunction can be orders of magnitudes larger at very low energies. Thus, we expect the "electron transfer channel" to be strongly favored below $4 \mathrm{eV}$; whereas at higher energies (e.g., at $9 \mathrm{eV}$ ) autoionization into vacuum should considerably increase. According to these decay channels, the present $\mathrm{H}^{-}$ yields could be produced via DEA (channel 3) and the $\mathrm{O}^{-}$and $\mathrm{OH}^{-}$yields via channel 1. This is consistent with previous results showing that in DNA, $\mathrm{H}^{-}$arises essentially from the bases, and with the present ones, which indicate that $\mathrm{O}^{-}$and $\mathrm{OH}^{-}$desorb following electron transfer to the phosphate group. The strong decrease in strand break and base release previously observed upon formation of an abasic site in single stranded DNA can be explained from decay into channel 2. Via this channel, even when LEE experiments are performed at energies higher than $3-5 \mathrm{eV}$, highly coherent electrons of energies lower than $3 \mathrm{eV}$ can be created by electronically inelastic scattering. In this case, the amplitude of the electron wave re-emitted at much lower energy is highly sensitive to the molecular arrangement of the oligonucleotide, a condition which strongly influences the branching ratios between electron decay in vacuum and within DNA. When electron coherence is destroyed within DNA (e.g., owing to molecular rearrangement following creation of an abasic site) electron emission in vacuum is considerably increased followed by a 
corresponding decrease in bond scission within DNA as observed in the experiments of Zheng et al. ${ }^{15}$

Thus, according to the mechanism suggested in Scheme 1, strong collective effects are not observed in the present experiments because to eject an anion from this film, as shown that direct DEA or DEA via electron transfer must occur via coreexcited resonances ${ }^{36}$ (i.e., at $\left.E_{0} \geq 4 \mathrm{eV}\right) .^{37,38}$

\section{Acknowledgements}

This work is financed by the Canadian Institutes of Health Research (CIHR). The authors would like to thank Mr P. Cloutier for technical support, Dr Y. Zheng for sample preparation and purification, Dr A. D. Bass for helpful suggestions and Prof. P. D. Burrow for constructive comments. S. Ptasińska would like to thank the CIHR for financial support in the form of a fellowship.

\section{References}

1 B. Boudaiffa, P. Cloutier, D. Hunting, M. A. Huels and L. Sanche, Science, 2000, 287, 1658.

2 For a review see L. Sanche, Eur. Phys. J. D, 2005, 35, 367.

3 X. Pan, P. Cloutier, D. Hunting and L. Sanche, Phys. Rev. Lett., 2003, 90, 208102-1.

4 X. Pan and L. Sanche, Phys. Rev. Lett., 2005, 94, 198104-1.

5 R. Barrios, P. Skurski and J. Simons, J. Phys. Chem., 2002, 106, 7991.

6 X. Li, M. D. Sevilla and L. Sanche, J. Am. Chem. Soc., 2003, 125, 13668.

7 Y. Zheng, P. Cloutier, D. J. Hunting, L. Sanche and J. R. Wagner, J. Am. Chem. Soc., 2005, 127, 16592.

8 Y. Zheng, P. Cloutier, D. J. Hunting, J. R. Wagner and L. Sanche, J. Chem. Phys., 2006, 124, 64710-1.

9 X. Li, L. Sanche and M. D. Sevilla, Radiat. Res., 2006, 165, 721.

10 J. Berdys, I. Anusiewicz, P. Skurski and J. Simons, J. Am. Chem. Soc., 2004, 126, 6441.

11 J. Berdys, P. Skurski and J. Simons, J. Phys. Chem. B, 2004, 108, 5800.

12 J. Berdys, I. Anusiewicz, P. Skurski and J. Simons, J. Phys. Chem. A, 2004, 108, 2999.

13 I. Dabkowska, J. Rak and M. Gutowski, Eur. Phys. J. D, 2005, 35, 429.

14 F. Martin, P. D. Burrow, Z. Cai, P. Cloutier, D. J. Hunting and L. Sanche, Phys. Rev. Lett., 2004, 93, 068101-1.

15 Y. Zheng, R. Wagner and L. Sanche, Phys. Rev. Lett., 2006, 96, 208101.
16 L. G. Caron and L. Sanche, Phys. Rev. A, 2005, 72, 032726-1.

17 L. G. Caron and L. Sanche, Phys. Rev. A, 2004, 70, 032719-1.

18 L. G. Caron and L. Sanche, Phys. Rev. Lett., 2003, 91, 113201-1.

19 M. A. Hervé du Penhoat, M. A. Huels, P. Cloutier, J.-P. Jay-Gerin and L. Sanche, J. Chem. Phys., 2001, 114, 5755.

20 H. Abdoul-Carime, P. Cloutier and L. Sanche, Radiat. Res., 2001, $155,625$.

21 G. D. Fasman, Handbook of Biochemistry and Molecular Biology, CRC Press, Boca Raton, FL, 3rd edn, 1995.

22 B. Boudaïffa, P. Cloutier, D. Hunting, M. A. Huels and L. Sanche, Radiat. Res., 2002, 157, 227.

23 J. Meesungnoen, J.-P. Jay-Gerin, A. Filali-Mouhim and S. Mankhetkorn, Radiat. Res., 2002, 158, 657.

24 K. Nagesha, J. Gamache, A. D. Bass and L. Sanche, Rev. Sci. Instrum., 1997, 68, 3883.

25 S. Ptasińska and L. Sanche, J. Chem. Phys., 2006, 125, 144713.

26 P. Mozejko, A. D. Bass, L. Parenteau and L. Sanche, J. Chem. Phys., 2004, 121, 10181.

27 H. Abdoul-Carime, J. Langer, M. A. Huels and E. Illenberger, Eur. Phys. J. D, 2005, 35, 399.

28 S. Denifl, S. Ptasinska, M. Cingel, S. Matejcik, P. Scheier and T. D. Märk, Chem. Phys. Lett., 2003, 377, 74.

29 S. Denifl, S. Ptasińska, M. Probst, J. Hrušák, P. Scheier and T. D. Märk, J. Phys. Chem. A, 2004, 108, 6562.

30 D. Huber, M. Beikircher, S. Denifl, F. Zappa, S. Matejcik, A. Bacher, V. Grill, T. D. Märk and P. Scheier, J. Chem. Phys., 2006, 125, 084304-1.

31 S. Ptasińska, S. Denifl, V. Grill, T. D. Märk, E. Illenberger and P. Scheier, Phys. Rev. Lett., 2005, 95, 093201.

32 S. Ptasińska, S. Denifl, B. Mróz, M. Probst, V. Grill, E. Illenberger, P. Scheier and T. D. Märk, J. Chem. Phys., 2005, 123, 124302-1.

33 S. Ptasińska, S. Denifl, V. Grill, T. D. Märk, P. Scheier, S. Gohlke, M. A. Huels and E. Illenberger, Angew. Chem., Int. Ed., 2005, 44, 1647.

34 C. König, J. Kopyra, I. Bald and E. Illenberger, Phys. Rev. Lett., 2006, 97, 018105-1.

35 X. Pan and L. Sanche, Chem. Phys. Lett., 2006, 421, 404.

36 H. Hotop, M.-W. Ruf, M. Allan and I. I. Fabrikant, Adv. At. Mol. Opt. Phys., 2003, 49, 85.

37 P. L. Lévesque, M. Michaud, W. Cho and L. Sanche, J. Chem. Phys., 2005, 122, 224704-1.

38 R. Abouaf, J. Pommier and H. Dunet, Chem. Phys. Lett., 2003, 381, 486.

39 L. G. Caron and L. Sanche, Phys. Rev. A, 2006, 73, 062707-1.

40 In condensed films core-excited (Feshbach) resonances can decay by electron emission below the vacuum level. In this case, the parent molecule can be left in an electronically excited state. See for example A. D. Bass, E. Vichnevetski and L. Sanche, Phys. Rev. B: Condens. Matter, 1999, 60, 14405. 